Article

\title{
Simple Synthesis Method and Characterizations of Aggregation-Free Cysteamine Capped PbS Quantum Dot
}

\author{
Daekyung Kang ${ }^{1}$, Mareddi Bharath Kumar ${ }^{1}$, Changhee Son ${ }^{1}$, Hongsik Park ${ }^{2}{ }^{\mathbb{D}}$ \\ and Jonghoo Park ${ }^{1, *}$ \\ 1 Department of Electrical Engineering, Kyungpook National University, Daehak-ro 80, Buk-gu, Daegu 41566, \\ Korea; kyoung528@knu.ac.kr (D.K.); 1992mbkr@gmail.com (M.B.K.); imeanx@gmail.com (C.S.) \\ 2 School of Electronic Engineering, Kyungpook National University, Daehak-ro 80, Buk-gu, Daegu 41566, \\ Korea; hpark@ee.knu.ac.kr \\ * Correspondence: jonghoopark@knu.ac.kr
}

Received: 25 September 2019; Accepted: 30 October 2019; Published: 1 November 2019

\begin{abstract}
Quantum dots have diverse chemical properties with different ligands attached on the surface. The cysteamine has been used as a ligand for various quantum dots because it has high solubility in water, and it facilitates binding of quantum dot and gold surface. However, the hydrogen bonds in cysteamine cause aggregation of the cysteamine capped quantum dots. In this study, we suggested a simple synthesis method of aggregation-free $\mathrm{PbS}$ quantum dot and analyzed the electric and optical properties of the synthesized quantum dot. This study on aggregation-free cysteamine capped quantum dots has the potential to develop advanced quantum dot-based sensor technologies, including biomedical imaging and environmental sensors.
\end{abstract}

Keywords: lead sulfide quantum dots; water-soluble quantum dots; cysteamine capped quantum dots; aggregation-free

\section{Introduction}

Over the past decade, quantum dots have attracted great attention in various research fields and industries because of their distinct electrical and optical properties from bulk materials. Three-dimensional quantum confinements are caused by particle size below Bohr's exciton radius effects on the energy band structure, which is deeply related to the electrical and optical properties of quantum dots [1,2]. Unlike in the bulk semiconductors, the dimension-dependent density of states has the shape of delta function in the quantum dots. This yields the absorption and emission spectra of quantum dots, with narrow full width half maximum (FWHM) centered at particular wavelength. The quantum dots have engineering advantages where the absorption and emission spectra can be adjusted by changing the synthesis conditions. These properties of quantum dots provide high wavelength-selectivity, accuracy, and sensitivity [3-6]. Therefore, quantum dots are considered one of the most promising materials in various applications, including optoelectronics [7,8], biosensors [9-14], energy harvesters [15-17], and quantum dot-based lasers [18,19].

In general, the core quantum dot consists of semiconductors, especially the compounds of II-VI or IV-VI materials. The PbS quantum dots, one of IV-VI quantum dots, have been studied in various research and industry fields because it absorbs and emits infrared (IR) radiation in the wavelength range of near and shortwave IR [20-22], which corresponds to the absorption energy range between 0.71 to $1.28 \mathrm{eV}$ [23]. The applications of PbS quantum dots with IR have been considered as one of the major subjects in research and industries, including optoelectronics and sensors [24-26]. 
While the core of a quantum dot determines the electrical and optical properties of the quantum dot, the ligand of the quantum dot generally characterizes chemical interactions with other materials including solvents, surfaces, and particles [27]. The development of ligands has made it possible to carry diverse chemical properties to quantum dots, making them more attractive to sensor applications $[28,29]$. In ligand groups comprising trioctylphosphine oxide (TOPO), trioctylphosphine (TOP), amines, and carboxyl acids, thiols are mainly used to synthesize gold-adherent quantum dots [30,31]. Since gold is one of the most used materials in biosensors [32-35], PbS quantum dots passivated with thiol-based ligands can have many advantages over other ligands for quantum dots in biosensor applications. Cysteamine, one of the thiols, is distinguished from other thiols because it is soluble in water, the most common and safe solvent. Thus, the various types of cysteamine capped quantum dots have been studied in the fields [36-38]. However, the synthesis of cysteamine capped PbS quantum dot has been considered difficult, due to the aggregation caused by hydrogen bonds between cysteamine molecules [39].

In this study, we describe a simple synthesis method of aggregation-free cysteamine capped $\mathrm{PbS}$ quantum dots. We employed sodium docusate as a stabilizing agent to maintain lead ions to be monomers, and 2-mercaptoethanesulfonate as an additional ligand to prevent the aggregation. The whole process was conducted in one pot and did not require any extra complex procedures. The size and lattice structure of the quantum dots were characterized by the transmission electron microscopy (TEM), and energy dispersive X-ray spectroscopy (EDS) confirmed that cysteamine and 2-mercaptoethanesulfonate were well-adhered to the surface of the quantum dot. The absorption characteristics of the quantum dot analyzed by the spectrophotometer shows high absorbance at the wavelength of $1450 \mathrm{~nm}$. The synthesized quantum dots maintain dispersion stability longer than one month.

\section{Materials and Methods}

\subsection{Reagents}

Lead(II) chloride (powder, 99\%) was purchased from Wako chemicals. Sodium sulfide hydrate $(\geq 60 \%)$, Docusate sodium salt (AOT) $(\geq 96 \%)$, Cysteamine ( $\geq 98 \%)$, Sodium 2-mercaptoethanesulfonate $(\geq 98 \%)$ were purchased from Sigma-Aldrich. Double distilled water (HLPC) was purchased from Daejung chemicals and metals.

\subsection{Synthesis of Aggregation-Free Cysteamine Capped PbS Quantum Dot}

Cysteamine capped $\mathrm{PbS}$ quantum dot was synthesized in aqueous solution with sodium docusate as a stabilizing agent. A $0.24 \mathrm{mmol}(0.105 \mathrm{~g})$ of sodium docusate salt was dissolved in $50 \mathrm{~mL}$ of double distilled water with stirring for $30 \mathrm{~min}$ on a magnetic stirrer, then a $3 \mathrm{~mL}$ of $0.04 \mathrm{M}$ lead chloride aqueous solution was added to the solution. Since the solubility of lead chloride in water $\left(10.8 \mathrm{~g} / \mathrm{L}, 20^{\circ} \mathrm{C}\right)$ is poor, it is recommended to keep the stock solution of lead precursors above $40^{\circ} \mathrm{C}$. After stirring for $10 \mathrm{~min}$, a $0.6 \mathrm{~mL}$ of $0.2 \mathrm{M}$ sodium sulfide aqueous solution was added dropwise to the solution and stirred for $10 \mathrm{~min}$. When the sulfur precursor was added, the color of the solution turned dark brown immediately. For ligand exchange, a $1 \mathrm{~mL}$ of $0.6 \mathrm{M}$ cysteamine aqueous solution was added into $\mathrm{PbS}$ quantum dot colloids and stirred for $10 \mathrm{~min}$. After adding the cysteamine to PbS quantum dots, the texture of suspension was changed. Lastly, a $1 \mathrm{~mL}$ of $0.6 \mathrm{M}$ 2-mercaptoethanesulfonate aqueous solution was dropped into cysteamine capped $\mathrm{PbS}$ quantum dot and stirred for $10 \mathrm{~min}$, to prevent the aggregation of quantum dots. A brief description of the synthesis method is shown in Figure 1. 


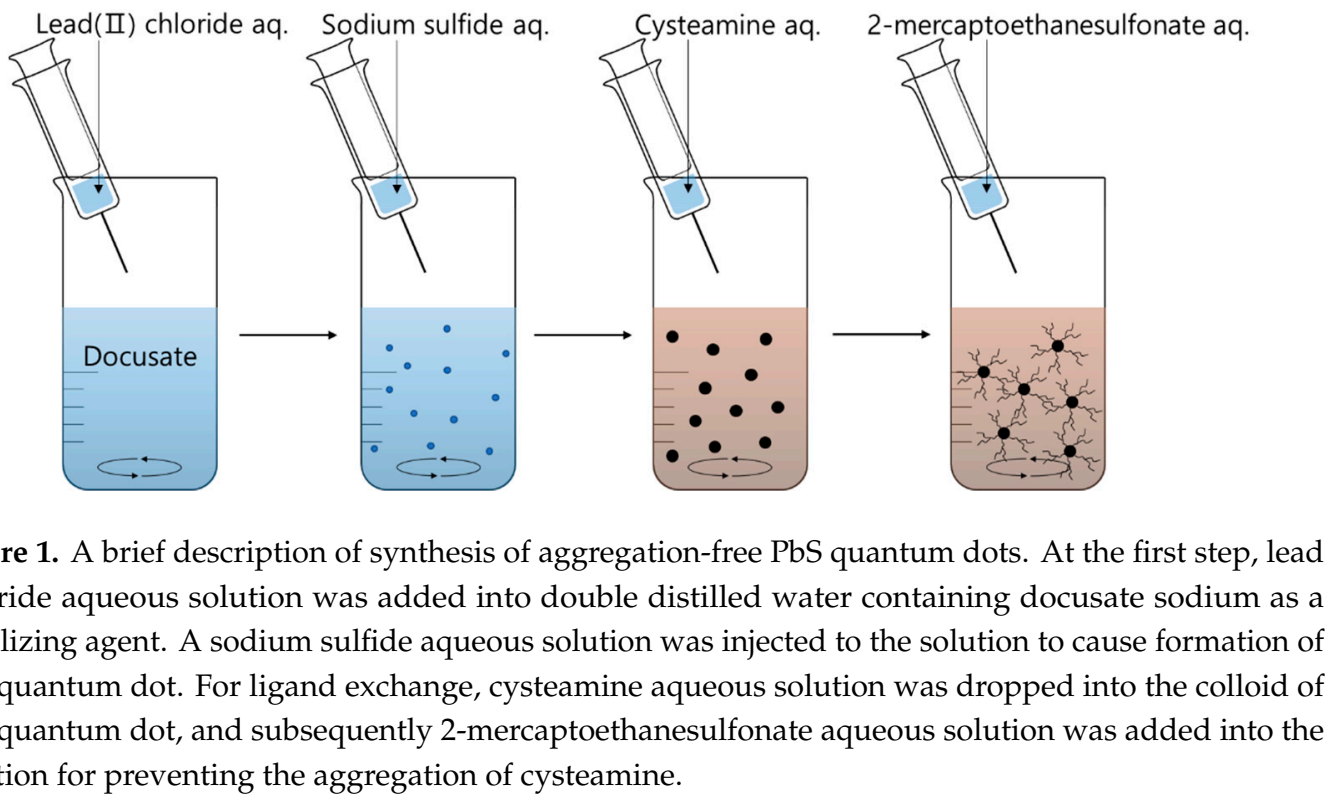

\subsection{Characterizations}

The TEM images and EDS measurements of aggregation-free cysteamine capped PbS quantum dot were captured by Titan G2 ChemiSTEM Cs Probe (FEI Company, Hillsboro, OR, USA). The synthesized quantum dots were placed on 200 meshes of formvar/copper TEM grid (TED PELLA, Inc., Redding, CA, USA) after dipping in colloid for less than $10 \mathrm{~s}$ and drying for over $10 \mathrm{~min}$ in atmosphere.

The infrared absorption spectrum of the colloidal PbS quantum dot was measured using transmission mode of Cary 5000 UV-Vis-NIR spectrophotometer (Agilent technologies, Inc., Santa Clara, CA, USA) in the form of liquids. Because water is not used for infrared spectroscopy due to its high infrared absorption, methanol which has low infrared absorption was used for the solvent. In order to disperse the synthesized quantum dots in methanol, the colloid was centrifuged for 90 min with 7000 of relative centrifugal force (RCF) in a centrifuge. Methanol was added to the precipitated quantum dots, and the colloid was sonicated for $5 \mathrm{~min}$.

The XRD (X-ray diffraction) measurement was conducted in form of powder. The synthesized quantum dot colloid was evaporated in room temperature. The result of XRD was obtained using EMPYREAN (PANanalytical, B.V., Almelo, The Netherlands).

\section{Results and Discussion}

The colloid of resulting cysteamine capped PbS quantum dot has been well-dissolved in double distilled water over a month. As it contained sodium docusate as a stabilizing agent, lead ion from lead chloride developed in the form of monomers. If the lead precursor is not dissolved as monomers, nuclear growth occurs before nuclei are created, which makes it difficult to control the size of the quantum dots. When the lead precursor is injected to water in which docusate is dissolved, the docusate molecules bind around the lead monomer, and the color of solution turns white. Subsequently, when the lead monomers react with the sulfur precursor, nuclei of PbS quantum dot start to form, and the size of the quantum dot gradually increases according to Ostwald ripening. The size of the quantum dots is usually proportional to the reaction time and temperature. After nuclear growth, cysteamine aqueous solution is added to induce ligand exchange on the surface of the quantum dot. However, since cysteamine has an amine group, there is a possibility that quantum dots may aggregate over time by forming hydrogen bonds in an aqueous solution, and it is necessary to additionally attach another ligand to prevent the aggregation. Since 2-mercaptoethanesulfonate is anionized in an aqueous solution, quantum dots can maintain dispersion stability even if they have cysteamine as a ligand, due to the electrical repulsion generated therefrom. The synthesized aggregation-free PbS quantum dots, 
as described above, it was confirmed that they did not aggregate and maintained dispersion stability even after one month. Although, some white and yellow precipitates were formed on the bottom of the solution. We attribute the white and yellow precipitates to lead compounds, including lead sulphate, lead carbonate, and lead oxide, caused by lead atoms not involved in quantum dot formation. Figure 2 shows the images of quantum dots as synthesized and after one month from synthesis.

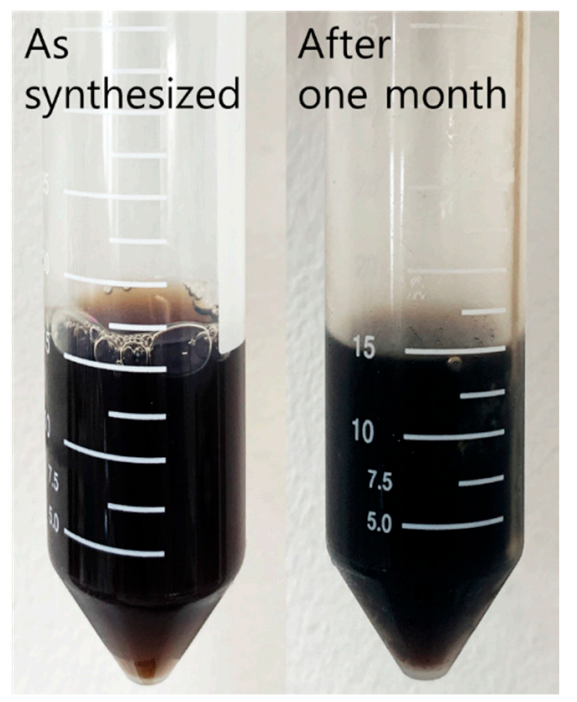

Figure 2. Image of cysteamine capped PbS quantum dot dispersed in water. (left) Image of quantum dot as synthesized, (right) Image of quantum dot one month after synthesis.

Figure 3a shows the TEM images of the synthesized quantum dot. The TEM images shows that the particles with particular diameter are evenly distributed on the grid. Figure $3 b$ shows a TEM image of quantum dots synthesized without adding 2-mercaptoethanesulfonate and severe aggregation of the quantum dots.

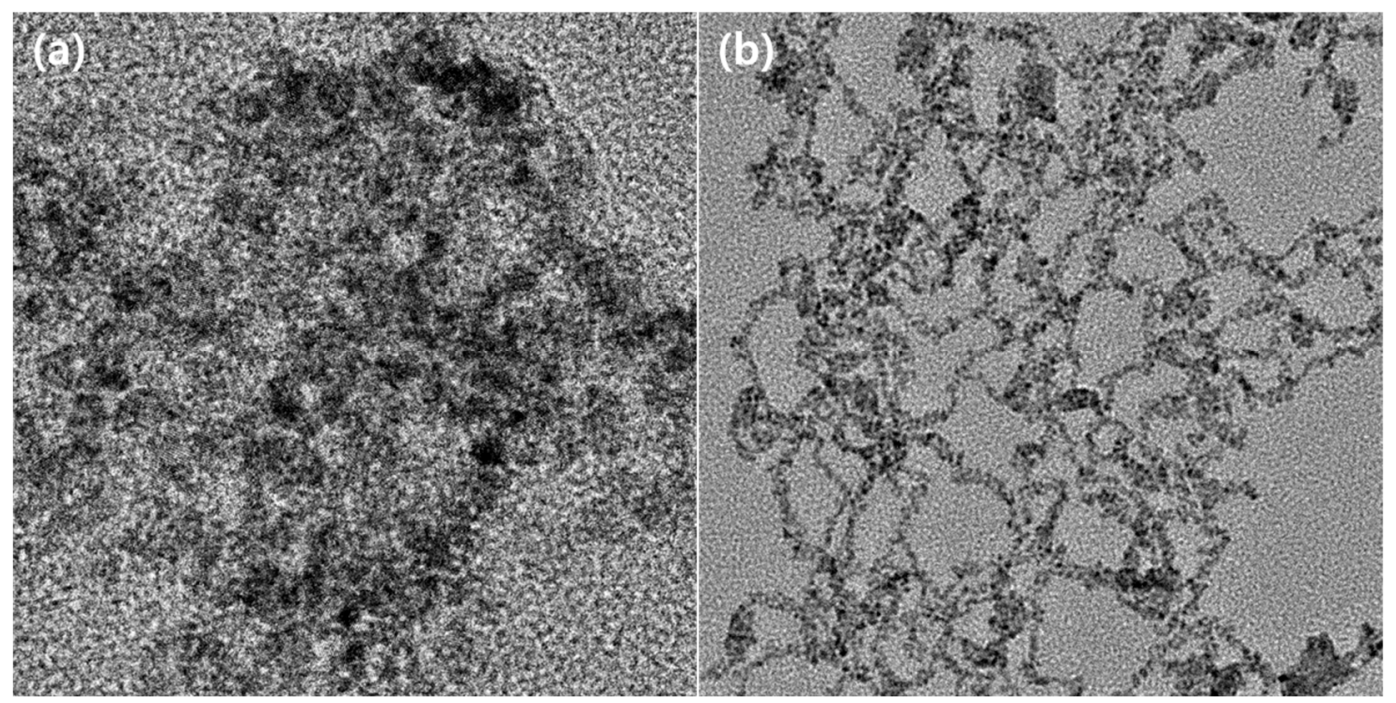

Figure 3. Transmission electron microscopy (TEM) images of cysteamine capped PbS quantum dots. (a) Image of cysteamine capped PbS quantum dot with 2-mercaptoethanesulfonate as additional ligand (b) Image of cysteamine capped $\mathrm{PbS}$ quantum dot without 2-mercaptoethanesulfonate. Unlike $(\mathbf{a}, \mathbf{b})$ shows the severe aggregation of $\mathrm{PbS}$ quantum dots caused from hydrogen bonds of cysteamine molecules. 
In Figure 3b, all particles are connected and aggregated with the hydrogen bond from amine functional groups of cysteamine. However, in Figure $3 a$, the separated particles are laid over the layers. From above, attaching 2-mercaptoethanesulfonate as an additional ligand was an efficient method to prevent the aggregation of cysteamine.

Figure 4 shows the TEM image of the synthesized quantum dots in a higher magnitude than in Figure 3. The particles in the image have three types of lattice structures which are PbS [111], $\mathrm{PbS}$ [200], and PbS [220]. The lattice constant of PbS is known as $5.963 \AA$. Thus, PbS quantum dots have the lattice plane spacings of $0.21 \mathrm{~nm}, 0.29 \mathrm{~nm}$, and $0.34 \mathrm{~nm}$ which correspond to PbS [220], PbS [200], and $\mathrm{PbS}$ [111], respectively.

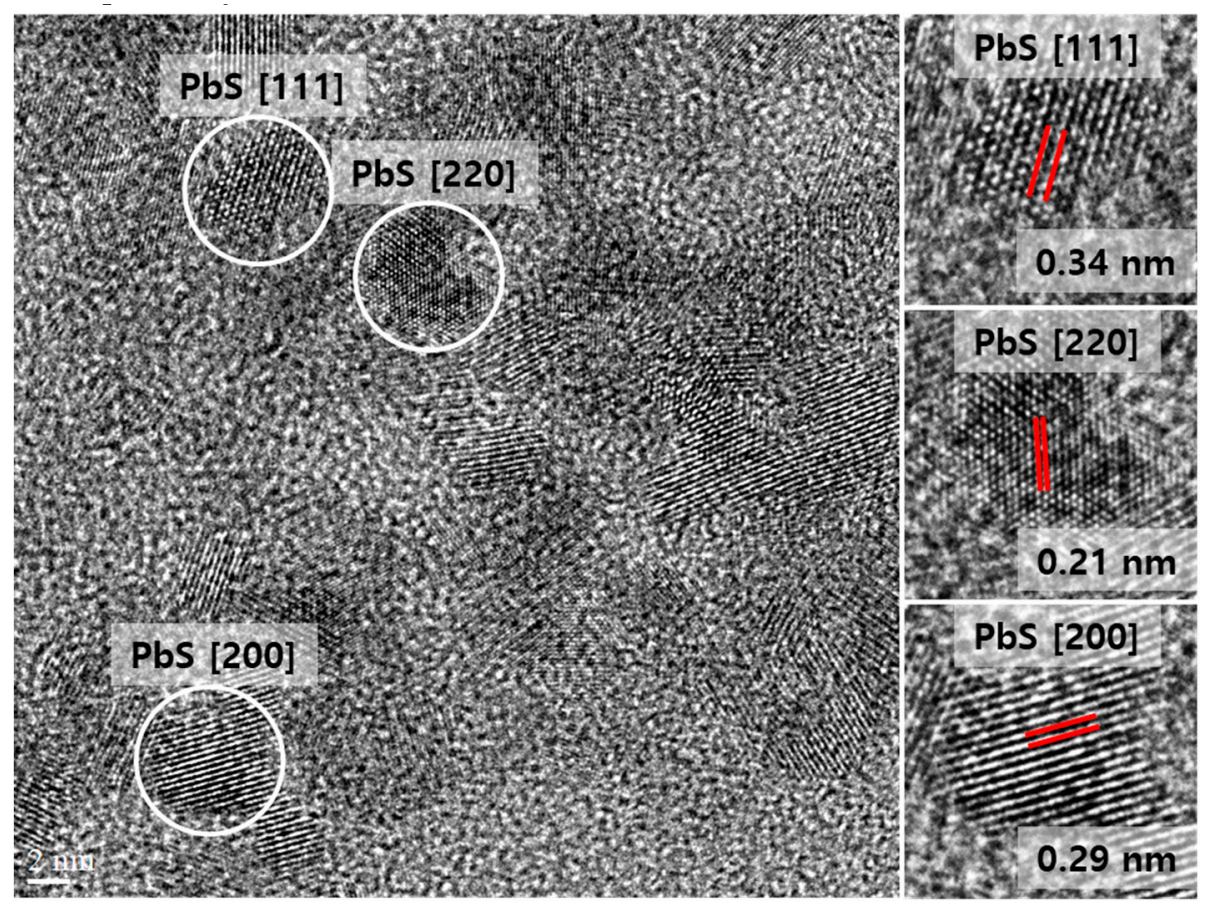

Figure 4. The TEM image of the synthesized quantum dots in a higher magnitude than in Figure 3. In the image, the particles are able to be classified by three types of lattice structures. The lattice plane spacing of the synthesized quantum dot are $0.21 \mathrm{~nm}, 0.29 \mathrm{~nm}$, and $0.34 \mathrm{~nm}$, which correspond to PbS [220], PbS [200], and PbS [111].

Figure 5 shows the TEM images of the synthesized quantum dot after one month. As the results in Figure 2, the synthesized quantum dot maintained the dispersion stability after one month. The TEM images of the synthesized quantum dot show that the particles are not aggregated after one month in the microscopic view. 


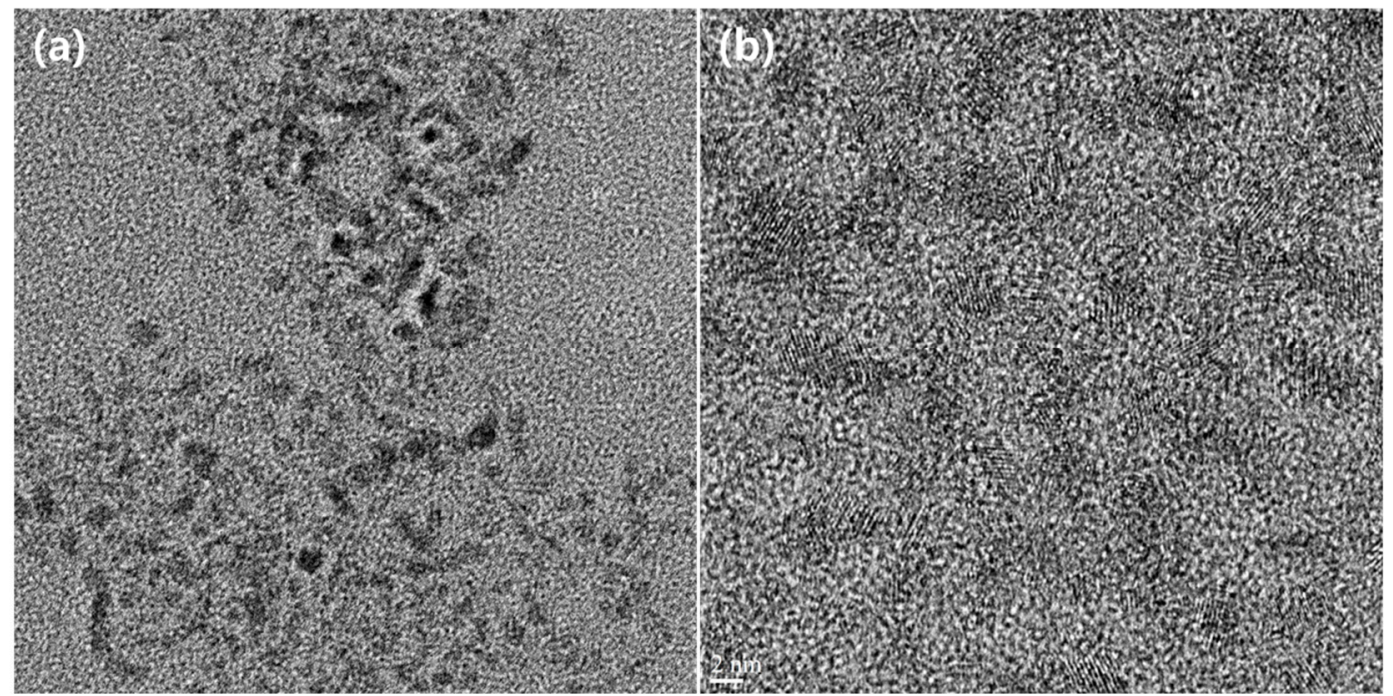

Figure 5. The TEM images of the synthesized quantum dot after one month. The particles are well-dispersed in water and are not aggregated in the images of TEM.

Figure 6 shows EDS results and it exhibits nitrogen and sodium atoms from the two ligands, indicating that the two ligands were well-attached to the surface of synthesized quantum dot.

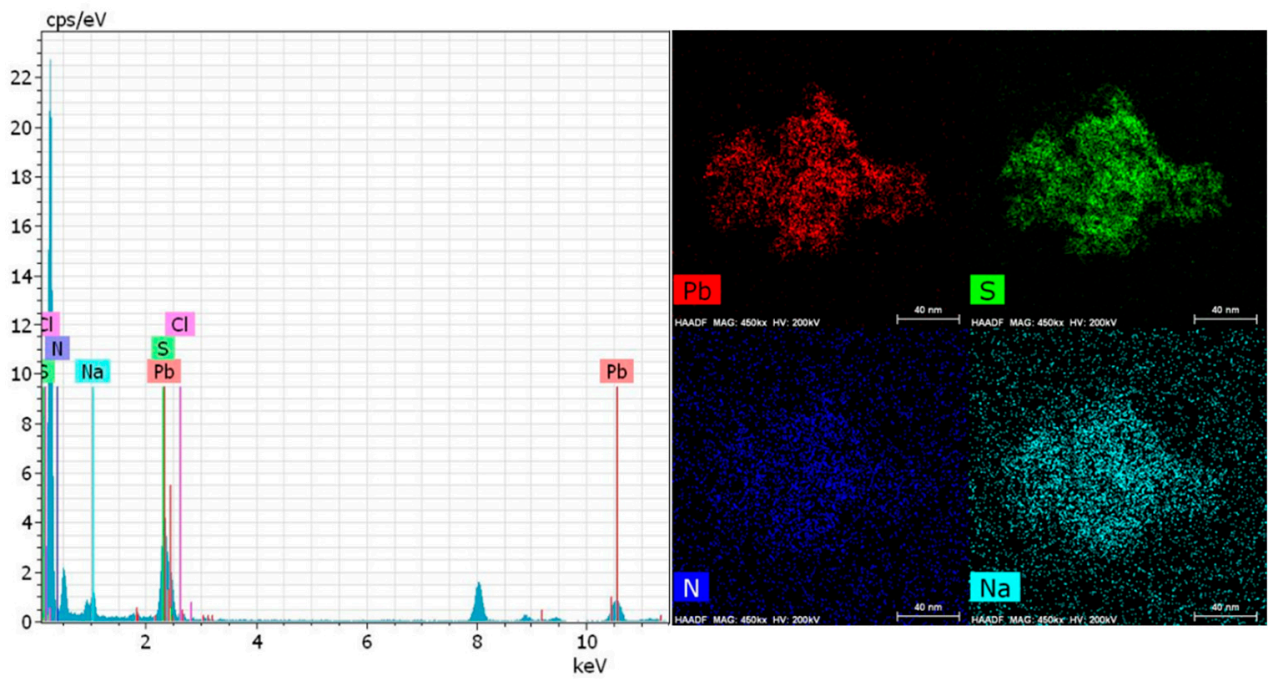

Figure 6. Energy dispersive X-ray spectroscopy (EDS) results of the aggregation-free cysteamine capped $\mathrm{PbS}$ quantum dot. The images show the presences of nitrogen (12 mol.\%) and sodium ( $21 \mathrm{~mol} . \%)$ atoms, indicating that the two ligands were attached to the surface of the PbS quantum dot.

Figure 7 shows the XRD results of the synthesized quantum dot. The highest peak of XRD is shown at PbS [111] and the next is PbS [220]. The intensity of PbS [200] is poor. Each points of peak correspond to the conventional PbS crystallinities [40].

Figure 8 demonstrates the absorption characteristics of synthesized cysteamine capped $\mathrm{PbS}$ quantum dot. The values used in Figure 5 were normalized between 0 to 1 . The absorbance of the synthesized quantum dot gradually decreases as the wavelength increases, and the absorbance starts to increase at the wavelength of $1360 \mathrm{~nm}$, reaches a peak at $1450 \mathrm{~nm}$, and then decreases again. The peak of absorbance is determined by the energy between conduction and valence band, which is reversely 
proportional to the size of the particle. According to Equation (1), the peak of absorbance is calculated to be $1430 \mathrm{~nm}$ as the average size of the synthesized quantum dot is $5.2 \mathrm{~nm}$ from TEM images.

$$
E_{0}=0.41+\frac{1}{0.0252 d^{2}+0.283 d}
$$

where $E_{0}$ is the absorption energy, and $d$ is the diameter of the quantum dot.

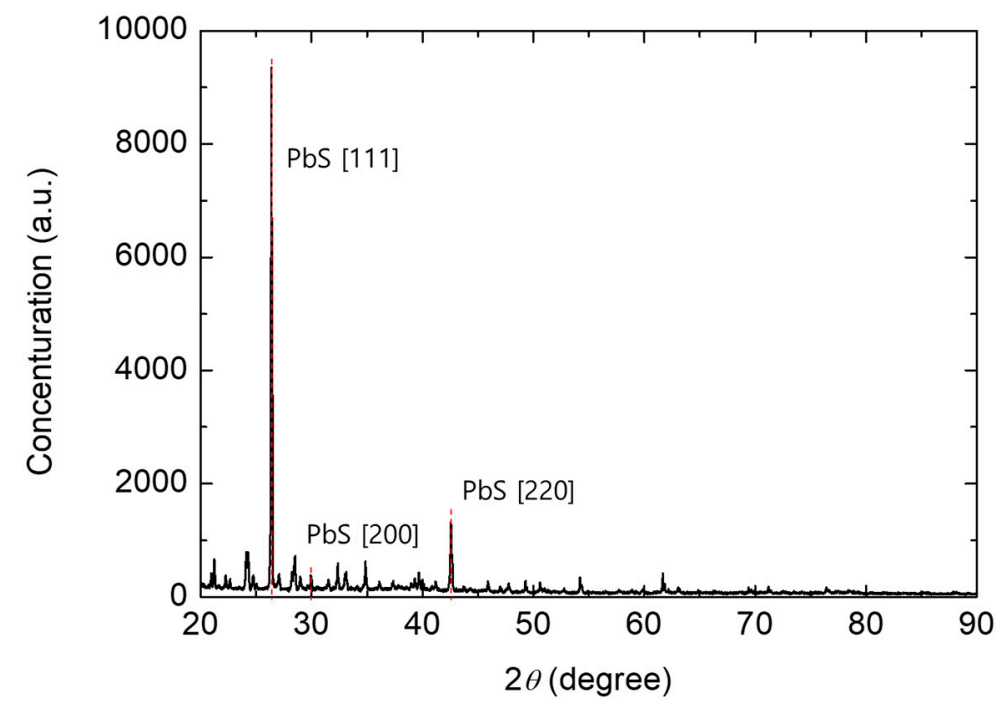

Figure 7. The X-ray diffraction (XRD) results of the synthesized quantum dot. In the graph, PbS [111] shows the highest intensity, and PbS [220] shows the next highest intensity. Each points of peak were detected at the specific points which correspond to the crystallinities of conventional $\mathrm{PbS}$.

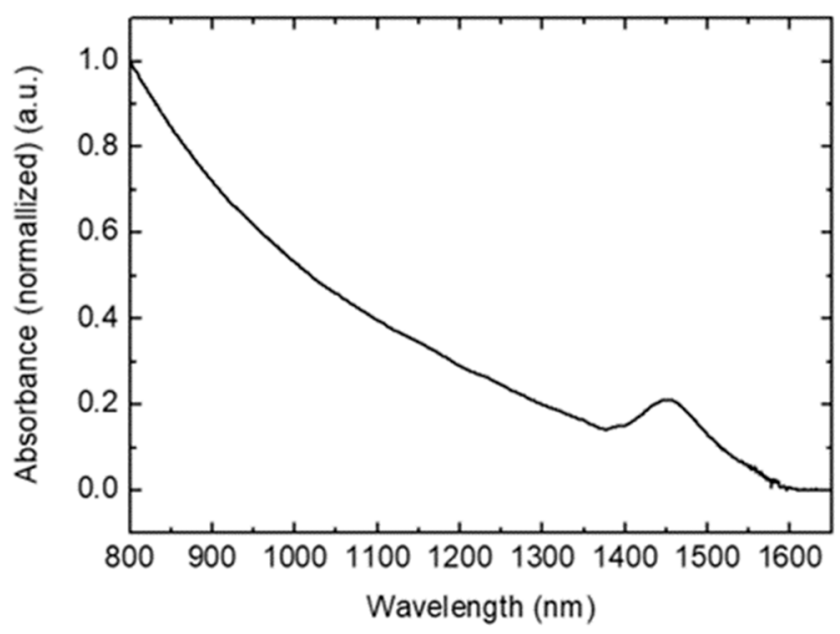

Figure 8. The absorbances of the aggregation-free cysteamine capped PbS quantum dot as synthesized. The absorbance of the quantum dot as synthesized is showing the peak wavelength of absorption at $1450 \mathrm{~nm}$ which is a good agreement with the theoretical works.

The measured peak wavelength of absorbance is $1450 \mathrm{~nm}$, which is a good agreement with the theoretical studies.

\section{Conclusions}

Among various ligands for quantum dots, cysteamine has attracted attention because of its water-solubility. The water-solubility of ligand offers great benefits in various quantum dot applications. However, the hydrogen bonds between cysteamine molecules prevent the aggregation-free cysteamine 
capped PbS quantum dots being synthesizing. In this study, we effectively solved this aggregation problem by additionally attaching 2-mercaptoethanesulfonate to the surface of the quantum dot to repel the attraction force from cysteamine. The structure, size, and IR absorption of the cysteamine capped $\mathrm{PbS}$ quantum dots were characterized by TEM, EDS, and spectrophotometer. We believe that this study on aggregation-free cysteamine quantum dots has a promising potential to develop advanced quantum dot-based sensor technologies, including biomedical imaging and environmental sensors.

Author Contributions: Methodology, D.K., M.B.K., C.S., H.P., and J.P.; validation, D.K., M.B.K., C.S., H.P., and J.P.; formal analysis, D.K., M.B.K., C.S., H.P., and J.P.; investigation, D.K.; data curation, D.K., and M.B.K.; writing-Original draft preparation, D.K., and J.P.; supervision, J.P.

Acknowledgments: This work was supported by the National Research Foundation of Korea (NRF) grant funded by the Korea government (MSIT) (2017R1A4A1015565) and NRF-2015R1D1A1A04060083.

Conflicts of Interest: The authors declare no conflict of interest.

\section{References}

1. Takagahara, T.; Takeda, K. Theory of the quantum confinement effect on excitons in quantum dots of indirect-gap materials. Phys. Rev. B 1992, 46, 15578. [CrossRef] [PubMed]

2. Alivisatos, A.P. Semiconductor clusters, nanocrystals, and quantum dots. Science 1996, 271, 933-937. [CrossRef]

3. Frasco, M.; Chaniotakis, N. Semiconductor quantum dots in chemical sensors and biosensors. Sensors 2009, 9, 7266-7286. [CrossRef] [PubMed]

4. Murphy, C.J. Optical sensing with quantum dots. Anal. Chem. 2002, 74, 520A-526A.

5. Chen, Y.; Rosenzweig, Z. Luminescent CdS quantum dots as selective ion probes. Anal. Chem. 2002, 74, 5132-5138. [CrossRef]

6. Medintz, I.L.; Uyeda, H.T.; Goldman, E.R.; Mattoussi, H. Quantum dot bioconjugates for imaging, labelling and sensing. Nat. Mater. 2005, 4, 435. [CrossRef]

7. Liu, H.; Gao, M.; McCaffrey, J.; Wasilewski, Z.; Fafard, S. Quantum dot infrared photodetectors. Appl. Phys. Lett. 2001, 78, 79-81. [CrossRef]

8. Sukhovatkin, V.; Hinds, S.; Brzozowski, L.; Sargent, E.H. Colloidal quantum-dot photodetectors exploiting multiexciton generation. Science 2009, 324, 1542-1544. [CrossRef]

9. Malic, L.; Sandros, M.G.; Tabrizian, M. Designed biointerface using near-infrared quantum dots for ultrasensitive surface plasmon resonance imaging biosensors. Anal. Chem. 2011, 83, 5222-5229. [CrossRef]

10. Yong, K.T.; Roy, I.; Ding, H.; Bergey, E.J.; Prasad, P.N. Biocompatible near-infrared quantum dots as ultrasensitive probes for long-term in vivo imaging applications. Small 2009, 5, 1997-2004. [CrossRef]

11. Hong, G.; Antaris, A.L.; Dai, H. Near-infrared fluorophores for biomedical imaging. Nat. Biomed. Eng. 2017, 1, 0010. [CrossRef]

12. Cai, W.; Shin, D.-W.; Chen, K.; Gheysens, O.; Cao, Q.; Wang, S.X.; Gambhir, S.S.; Chen, X. Peptide-labeled near-infrared quantum dots for imaging tumor vasculature in living subjects. Nano Lett. 2006, 6, 669-676. [CrossRef]

13. Nakane, Y.; Tsukasaki, Y.; Sakata, T.; Yasuda, H.; Jin, T. Aqueous synthesis of glutathione-coated PbS quantum dots with tunable emission for non-invasive fluorescence imaging in the second near-infrared biological window (1000-1400 nm). Chem. Commun. 2013, 49, 7584-7586. [CrossRef] [PubMed]

14. Jin, T.; Imamura, Y. Applications of highly bright pbs quantum dots to non-invasive near-infrared fluorescence imaging in the second optical window. ECS J. Solid State Sci. Technol. 2016, 5, R3138-R3145. [CrossRef]

15. Robel, I.; Subramanian, V.; Kuno, M.; Kamat, P.V. Quantum dot solar cells. Harvesting light energy with CdSe nanocrystals molecularly linked to mesoscopic TiO2 films. J. Am. Chem. Soc. 2006, 128, 2385-2393. [CrossRef]

16. Kamat, P.V. Quantum dot solar cells. Semiconductor nanocrystals as light harvesters. J. Phys. Chem. C 2008, 112, 18737-18753. [CrossRef]

17. Sargent, E.H. Colloidal quantum dot solar cells. Nat. Photonics 2012, 6, 133. [CrossRef]

18. Wang, Y.; Li, X.; Song, J.; Xiao, L.; Zeng, H.; Sun, H. All-inorganic colloidal perovskite quantum dots: A new class of lasing materials with favorable characteristics. Adv. Mater. 2015, 27, 7101-7108. [CrossRef] 
19. Wang, Y.; Sun, H. Advances and prospects of lasers developed from colloidal semiconductor nanostructures. Prog. Quantum Electron. 2018, 60, 1-29. [CrossRef]

20. McDonald, S.A.; Konstantatos, G.; Zhang, S.; Cyr, P.W.; Klem, E.J.; Levina, L.; Sargent, E.H. Solution-processed $\mathrm{PbS}$ quantum dot infrared photodetectors and photovoltaics. Nat. Mater. 2005, 4, 138. [CrossRef]

21. Sargent, E.H. Infrared quantum dots. Adv. Mater. 2005, 17, 515-522. [CrossRef]

22. De Iacovo, A.; Venettacci, C.; Colace, L.; Scopa, L.; Foglia, S. PbS Colloidal Quantum Dot Photodetectors operating in the near infrared. Sci. Rep. 2016, 6, 37913. [CrossRef] [PubMed]

23. Moreels, I.; Lambert, K.; Smeets, D.; De Muynck, D.; Nollet, T.; Martins, J.C.; Vanhaecke, F.; Vantomme, A.; Delerue, C.; Allan, G. Size-dependent optical properties of colloidal PbS quantum dots. ACS Nano 2009, 3, 3023-3030. [CrossRef] [PubMed]

24. Litvin, A.; Martynenko, I.; Purcell-Milton, F.; Baranov, A.; Fedorov, A.; Gun'Ko, Y. Colloidal quantum dots for optoelectronics. J. Mater. Chem. A 2017, 5, 13252-13275. [CrossRef]

25. Kufer, D.; Nikitskiy, I.; Lasanta, T.; Navickaite, G.; Koppens, F.H.; Konstantatos, G. Hybrid 2D-0D MoS2-PbS quantum dot photodetectors. Adv. Mater. 2015, 27, 176-180. [CrossRef] [PubMed]

26. Sun, Z.; Liu, Z.; Li, J.; Tai, G.a.; Lau, S.P.; Yan, F. Infrared photodetectors based on CVD-grown graphene and $\mathrm{PbS}$ quantum dots with ultrahigh responsivity. Adv. Mater. 2012, 24, 5878-5883. [CrossRef] [PubMed]

27. Green, M. The nature of quantum dot capping ligands. J. Mater. Chem. 2010, 20, 5797-5809. [CrossRef]

28. Lidke, D.S.; Nagy, P.; Heintzmann, R.; Arndt-Jovin, D.J.; Post, J.N.; Grecco, H.E.; Jares-Erijman, E.A.; Jovin, T.M. Quantum dot ligands provide new insights into erbB/HER receptor-mediated signal transduction. Nat. Biotechnol. 2004, 22, 198. [CrossRef]

29. Tang, J.; Kemp, K.W.; Hoogland, S.; Jeong, K.S.; Liu, H.; Levina, L.; Furukawa, M.; Wang, X.; Debnath, R.; Cha, D. Colloidal-quantum-dot photovoltaics using atomic-ligand passivation. Nat. Mater. 2011, 10, 765. [CrossRef]

30. Woehrle, G.H.; Brown, L.O.; Hutchison, J.E. Thiol-functionalized, 1.5-nm gold nanoparticles through ligand exchange reactions: Scope and mechanism of ligand exchange. J. Am. Chem. Soc. 2005, 127, 2172-2183. [CrossRef]

31. Brust, M.; Fink, J.; Bethell, D.; Schiffrin, D.; Kiely, C. Synthesis and reactions of functionalised gold nanoparticles. J. Chem. Soc. Chem. Commun. 1995, 16, 1655-1656. [CrossRef]

32. Liu, J.; Lu, Y. Adenosine-dependent assembly of aptazyme-functionalized gold nanoparticles and its application as a colorimetric biosensor. Anal. Chem. 2004, 76, 1627-1632. [CrossRef] [PubMed]

33. Yu, C.; Irudayaraj, J. Multiplex biosensor using gold nanorods. Anal. Chem. 2007, 79, 572-579. [CrossRef] [PubMed]

34. Luo, X.-L.; Xu, J.-J.; Du, Y.; Chen, H.-Y. A glucose biosensor based on chitosan-glucose oxidase-gold nanoparticles biocomposite formed by one-step electrodeposition. Anal. Biochem. 2004, 334, 284-289. [CrossRef] [PubMed]

35. Liu, J.; Lu, Y. A colorimetric lead biosensor using DNAzyme-directed assembly of gold nanoparticles. J. Am. Chem. Soc. 2003, 125, 6642-6643. [CrossRef] [PubMed]

36. Pei, J.; Zhu, H.; Wang, X.; Zhang, H.; Yang, X. Synthesis of cysteamine-coated CdTe quantum dots and its application in mercury (II) detection. Anal. Chim. Acta 2012, 757, 63-68. [CrossRef] [PubMed]

37. Noipa, T.; Tuntulani, T.; Ngeontae, W. Cu2+-modulated cysteamine-capped CdS quantum dots as a turn-on fluorescence sensor for cyanide recognition. Talanta 2013, 105, 320-326. [CrossRef]

38. Yaraki, M.T.; Tayebi, M.; Ahmadieh, M.; Tahriri, M.; Vashaee, D.; Tayebi, L. Synthesis and optical properties of cysteamine-capped ZnS quantum dots for aflatoxin quantification. J. Alloys Compd. 2017, 690, 749-758. [CrossRef]

39. Liu, J.; Yang, X.; Wang, K.; He, X.; Wang, Q.; Huang, J.; Liu, Y. Aggregation control of quantum dots through ion-mediated hydrogen bonding shielding. ACS Nano 2012, 6, 4973-4983. [CrossRef]

40. Kumar, D.; Agarwal, G.; Tripathi, B.; Vyas, D.; Kulshrestha, V. Characterization of PbS nanoparticles synthesized by chemical bath deposition. J. Alloys Compd. 2009, 484, 463-466. [CrossRef]

(C) 2019 by the authors. Licensee MDPI, Basel, Switzerland. This article is an open access article distributed under the terms and conditions of the Creative Commons Attribution (CC BY) license (http://creativecommons.org/licenses/by/4.0/). 